\title{
Commensurate structural modulation in the charge- and orbitally ordered phase of the quadruple perovskite $\left(\mathrm{NaMn}_{3}\right) \mathrm{Mn}_{4} \mathrm{O}_{12}$
}

\author{
A. Prodi,,${ }^{1, *}$ A. Daoud-Aladine, ${ }^{2}$ F. Gozzo, ${ }^{1, \dagger}$ B. Schmitt, ${ }^{1}$ O. Lebedev,${ }^{3}$ G. van Tendeloo, ${ }^{3}$ E. Gilioli, ${ }^{4}$ F. Bolzoni,${ }^{4}$ \\ H. Aruga-Katori, ${ }^{5, \ddagger}$ H. Takagi,,${ }^{5, \S}$ M. Marezio, ${ }^{6}$ and A. Gauzzi ${ }^{7}$ \\ ${ }^{1}$ Swiss Light Source, Paul Scherrer Institut, 5232 Villigen, Switzerland \\ ${ }_{2}^{2}$ ISIS Facility, STFC Rutherford Appleton Laboratory, Chilton, Didcot, Oxfordshire OX11 OQX, United Kingdom \\ ${ }^{3}$ EMAT, University of Antwerp, 2020 Antwerp, Belgium \\ ${ }^{4}$ Istituto dei Materiali per Elettronica e Magnetismo, CNR, Area delle Scienze, 43100 Parma, Italy \\ ${ }^{5}$ RIKEN (The Institute of Physical and Chemical Research), 2-1 Hirosawa, Wako, Saitama 351-0198, Japan \\ ${ }^{6}$ CRETA-CNRS, 25 avenue des Martyrs, 38042 Grenoble, France \\ ${ }^{7}$ IMPMC, UPMC-Sorbonne Universités, CNRS, MNHN, IRD, 4, place Jussieu, 75005 Paris, France \\ (Received 26 August 2014; revised manuscript received 2 October 2014; published 10 November 2014)
}

\begin{abstract}
By means of synchrotron x-ray and electron diffraction, we studied the structural changes at the charge order transition $T_{\mathrm{CO}}=176 \mathrm{~K}$ in the mixed-valence quadruple perovskite $\left(\mathrm{NaMn}_{3}\right) \mathrm{Mn}_{4} \mathrm{O}_{12}$. Below $T_{\mathrm{CO}}$ we find satellite peaks indicating a commensurate structural modulation with the same propagation vector $\mathbf{q}=(1 / 2,0,-1 / 2)$ of the $\mathrm{CE}$ magnetic structure that orders at low temperatures, similarly to the case of simple perovskites such as $\mathrm{La}_{0.5} \mathrm{Ca}_{0.5} \mathrm{MnO}_{3}$. In the present case, the modulated structure, together with the observation of a large entropy change at $T_{\mathrm{CO}}$, gives evidence of a rare case of full $\mathrm{Mn}^{3+} / \mathrm{Mn}^{4+}$ charge and orbital order, consistent with the Goodenough-Kanamori model.
\end{abstract}

DOI: 10.1103/PhysRevB.90.180101

PACS number(s): 71.70.Ej, 61.05.C-, 75.25.Dk

Quadruple perovskites $A A_{3}^{\prime} B_{4} \mathrm{O}_{12}$ have recently attracted a great deal of interest for their pronounced charge, spin, and orbital orderings [1-3] and for their promising dielectric [4-6] and multiferroic properties [7-9]. These compounds share with simple perovskites $\mathrm{ABO}_{3}$ a similar pseudocubic network of corner-sharing $\mathrm{BO}_{6}$ octahedra but differ in an unusually large tilt of these octahedra stabilized by the Jahn-Teller distortion of the $A^{\prime}$ site. This significantly reduces the electron hopping and alters the exchange interaction between neighboring $B$ sites. The large tilt reduces to four the number of first nearest oxygen atoms of the $A^{\prime}$ site, thus preventing oxygen vacancies [9]. The disorder inherent in chemically substituted perovskites, such as the prototype system $\mathrm{La}_{1-x} \mathrm{Sr}_{x} \mathrm{MnO}_{3}$ [10], is also absent, for mixed valence of the octahedral $B$ site is achieved by varying separately the valence of the $A$ and $A^{\prime}$ sites [11].

In view of these considerations, quadruple perovskites are a model system for studying novel ground states in transition metal oxides in the absence of disorder. The case of $\left(\mathrm{NaMn}_{3}\right) \mathrm{Mn}_{4} \mathrm{O}_{12}$ [11] is interesting owing to the +3.5 average valence of the $\mathrm{Mn}$ ions in the octahedral $B$ site corresponding to a 0.5 filling of the $e_{g} d_{x^{2}-y^{2}}-d_{3 z^{2}-r^{2}}$ doublet. In this compound, shown in Fig. 1, some of us previously reported at $T_{\mathrm{CO}}=176 \mathrm{~K}$ an almost full checkerboard-type $\mathrm{Mn}^{3+} / \mathrm{Mn}^{4+}$ charge order (CO) of the $B$ sites in the $a c$ plane, concomitant to a cubic $\operatorname{Im} \overline{3}$ to monoclinic $I 2 / \mathrm{m}$ phase transition driven by a pronounced Jahn-Teller distortion of

*Corresponding author: prodi@esrf.fr; Present address: ESRF, Grenoble, France.

${ }^{\dagger}$ Present address: Excelsus Structural Solutions SPRL, 1150 Bruxelles, Belgium.

${ }^{\ddagger}$ Present address: Institute of Engineering, Tokyo University of Agriculture and Technology, Koganei, Tokyo 184-8588, Japan.

${ }^{\S}$ Present address: Department of Physics, University of Tokyo Hongo, Bunkyo-ku, Tokyo 113-0033. the $\mathrm{Mn}^{3+} \mathrm{O}_{6}$ octahedra [1]. Powder neutron diffraction data indicated a compression of the octahedra along the $b$ axis, instead of the zigzag pattern of elongated octahedra in the ac plane, leading to a zigzag $d_{3 z^{2}-r^{2}}$ orbital order (OO), characteristic of simple perovskites such as $\mathrm{La}_{0.5} \mathrm{Ca}_{0.5} \mathrm{MnO}_{3}$ and related compounds at the same filling level. On the other hand, a compression would imply the occupancy of the $d_{x^{2}-y^{2}}$ orbital and thus an isotropic exchange interaction between neighboring $\operatorname{Mn}(B)$ ions within the $a c$ plane. According to the Goodenough-Kanamori-Anderson (GKA) rules [12], this type of interaction would not be compatible with the CE magnetic structure observed at low temperatures [1]. A very recent $a b$ initio study [13] has addressed this point by predicting for $\left(\mathrm{NaMn}_{3}\right) \mathrm{Mn}_{4} \mathrm{O}_{12}$ the same pattern of elongated octahedra found previously in $\mathrm{La}_{0.5} \mathrm{Ca}_{0.5} \mathrm{MnO}_{3}$.

In order to clarify this point, in this Rapid Communication we reinvestigate experimentally the low-temperature crystal structure of $\left(\mathrm{NaMn}_{3}\right) \mathrm{Mn}_{4} \mathrm{O}_{12}$ by means of transmission electron and high-resolution powder synchrotron $\mathrm{x}$-ray diffraction.

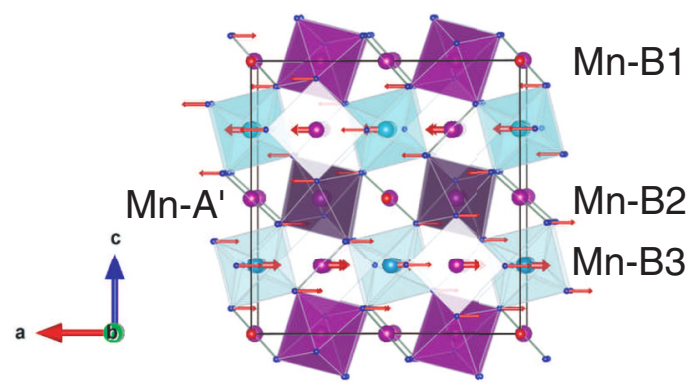

FIG. 1. (Color online) The monoclinic $C 2 / m$ structure of $\left(\mathrm{NaMn}_{3}\right) \mathrm{Mn}_{4} \mathrm{O}_{12}$ in the charge-ordered phase below $T_{\mathrm{CO}} . B 1-3$ indicate the nonequivalent $B$ octahedral sites of the $\mathrm{Mn}$ ions. $\mathrm{Mn}^{3+} \mathrm{O}_{6}$ and $\mathrm{Mn}^{4+} \mathrm{O}_{6}$ octahedra are shaded in purple and light blue, respectively. Arrows describe the displacement of the latter octahedra in the modulated structure. 


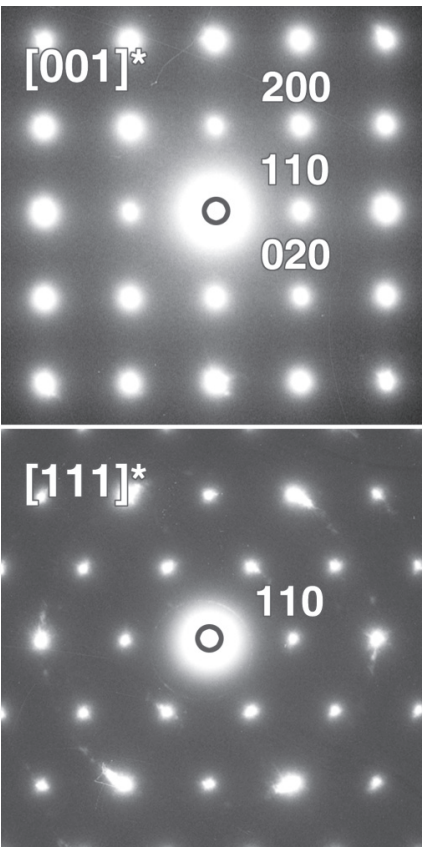

(a) RT

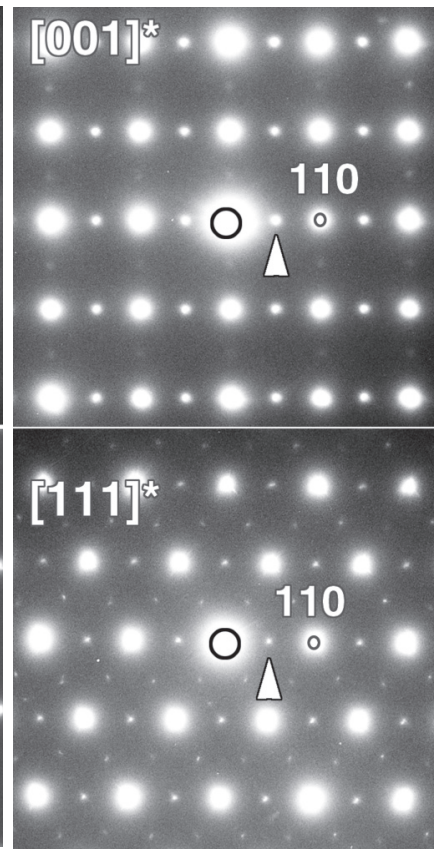

(b) $92 \mathrm{~K}$

FIG. 2. [001] $]^{*}$ and $[111]^{*}$ zone-axis electron diffraction patterns obtained (a) above and (b) below the charge-ordering transition in $\left(\mathrm{NaMn}_{3}\right) \mathrm{Mn}_{4} \mathrm{O}_{12}$. Arrows indicate the direction of the modulation and indices refer to the cubic $\operatorname{Im} \overline{3}$ lattice.

We find a commensurate structural modulation concomitant to the $\mathrm{CO}$ transition that confirms the above theoretical prediction and resolves the aforementioned discrepancy between structural distortion and magnetic order by providing a consistent description of the $\mathrm{CO}-\mathrm{OO}$ order at 0.5 doping in perovskitelike manganites within the GKA model of superexchange.

For this experiment, we used $\left(\mathrm{NaMn}_{3}\right) \mathrm{Mn}_{4} \mathrm{O}_{12}$ powders synthesized under high pressure, as described elsewhere [14]. $\mathrm{X}$-ray diffraction measurements confirmed that the sample is almost single phase with $\lesssim 4 \%$ weight of $\mathrm{Mn}_{2} \mathrm{O}_{3}$ impurities. Electron diffraction experiments were performed at room temperature and at $92 \mathrm{~K}$ using a Philips CM20 microscope operating at $200 \mathrm{kV}$ equipped with a low-temperature sample stage. High-resolution lattice images detected the presence of grain boundaries but no sign of point defects or microtwins. Thus, the electron diffraction patterns could be recorded on single-domain crystallites. In Fig. 2, we compare the patterns along the $[001]^{*}$ and $[111]^{*}$ zone axes at room temperature and below the structural transition at $92 \mathrm{~K}$ for one representative crystallite. The $92 \mathrm{~K}$ pattern displays extra spots along the [110] direction, which indicates a commensurate modulation with a propagation vector $\mathbf{q}=(1 / 2,0,-1 / 2)$ corresponding to a doubling of the cell along the $a$ and $c$ axes. The same commensurate $\mathbf{q}$ was found in several crystallites.

High-resolution synchrotron $\mathrm{x}$-ray powder diffractograms were collected at several temperatures at the X04SA Beamline [15] of the Swiss Light Source in Villigen using a wavelength $\lambda=0.708611(1) \AA$. A Rietveld refinement confirms the space groups and the lattice parameters reported in the previous neutron diffraction study, specifically the cubic $\operatorname{Im} \overline{3}$ to monoclinic $I 2 / m$ symmetry lowering at $T_{\mathrm{CO}}[1]$. In the X-ray diffractogram

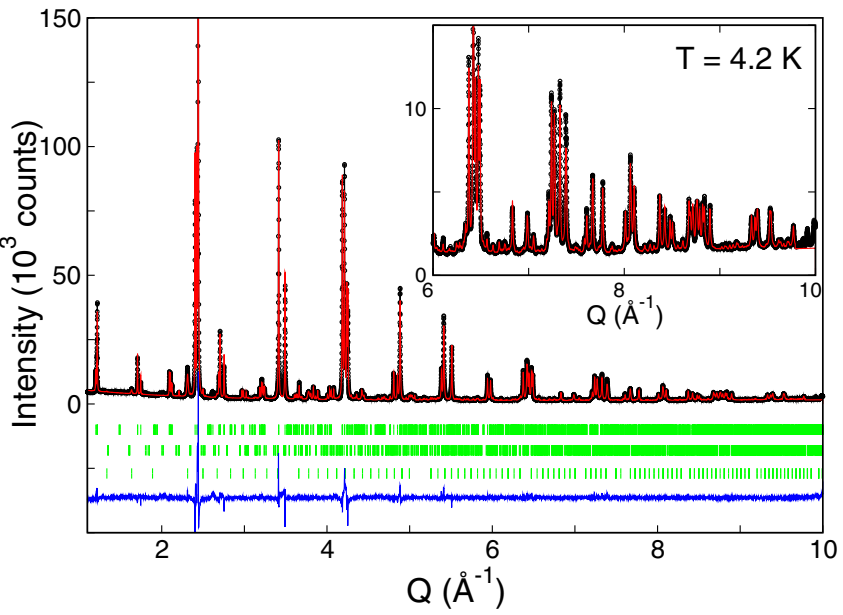

FIG. 3. (Color online) Full-pattern synchrotron x-ray Rietveld refinement for $\left(\mathrm{NaMn}_{3}\right) \mathrm{Mn}_{4} \mathrm{O}_{12}$ at $T=4.2 \mathrm{~K}$. The three rows of green ticks mark, from top to bottom, the position of the Bragg peaks of the main $I 2 / \mathrm{m}$ structure, of the superlattice, and of the $\mathrm{Mn}_{2} \mathrm{O}_{3}$ impurity. The blue line indicates the difference between the observed and calculated profile. The intensity of the strongest reflections was truncated to emphasize the details of the weaker reflections.

taken at $T=4.2 \mathrm{~K}$ (see Fig. 3), one notes superlattice (SL) reflections indexed with the commensurate propagation vector $\mathbf{q}=(1 / 2,0,-1 / 2)$, consistent with the electron diffraction data. We observed a dozen SL reflections that do not overlap with those of the main structure. Their intensities are a few percent of the most intense peaks, while their full width at half maxima (FWHM) indicate a correlation length of the modulation of $\approx 1000 \AA$. The temperature evolution of the integrated intensity of the $\left(\frac{3}{2}, 3, \frac{3}{2}\right)$ reflection shown in Fig. 4(a) shows that the modulation occurs at the cubic-monoclinic transition at $T_{\mathrm{CO}}$.

Hereafter we present a model of structural modulation of the $I 2 / m$ structure previously reported [1] which explains the above observations. As in the case of doped manganites, e.g., $\mathrm{La}_{0.5} \mathrm{Ca}_{0.5} \mathrm{MnO}_{3}$, the modulation has the same periodicity of the $\mathrm{CE}$ magnetic structure observed below $T_{N, B}=125 \mathrm{~K}$, which indicates a simultaneous charge and orbital order. The loss of translational symmetry caused by the modulation implies a lowering of the $I 2 / m$ symmetry. The group-subgroup path followed by the phase transition corresponds to a symmetry lowering within the double cubic perovskite cell from cubic Im $\overline{3} \rightarrow$ orthorhombic $I m m m \rightarrow$ monoclinic $I 2 / m$, involving $k=(000)$ modes and the additional modulation. Such a phase transition is required to be of first order in Landau theory [16], consistent with the discontinuity of the lattice parameters [1] and of the entropy at $T_{\mathrm{CO}}$, as seen in Fig. 4(a).

The symmetry of the modulated displacement follows the representation $A 1+$ of the small group of $I 2 / m$ with $k=\left(\frac{1}{2}, 0,-\frac{1}{2}\right)$, leading to a supercell with $I 2 / m$ symmetry (space group n. 12) and $\mathbf{a}_{s}=\mathbf{2 a}, \mathbf{b}_{s}=\mathbf{b}$, and $\mathbf{c}_{s}=-\mathbf{a}+\mathbf{c}$. We choose the equivalent $C$-centered monoclinic supercell in the $C 2 / m$ symmetry with basis vectors $\mathbf{a}_{s}=\mathbf{a}+\mathbf{c}, \mathbf{b}_{s}=\mathbf{b}$, and $\mathbf{c}_{s}=-\mathbf{a}+\mathbf{c}$, where $\mathbf{a}, \mathbf{b}$, and $\mathbf{c}$ are the basis vectors of the nonmodulated $I 2 / \mathrm{m}$ monoclinic structure and the subscript $s$ stays for the supercell. 


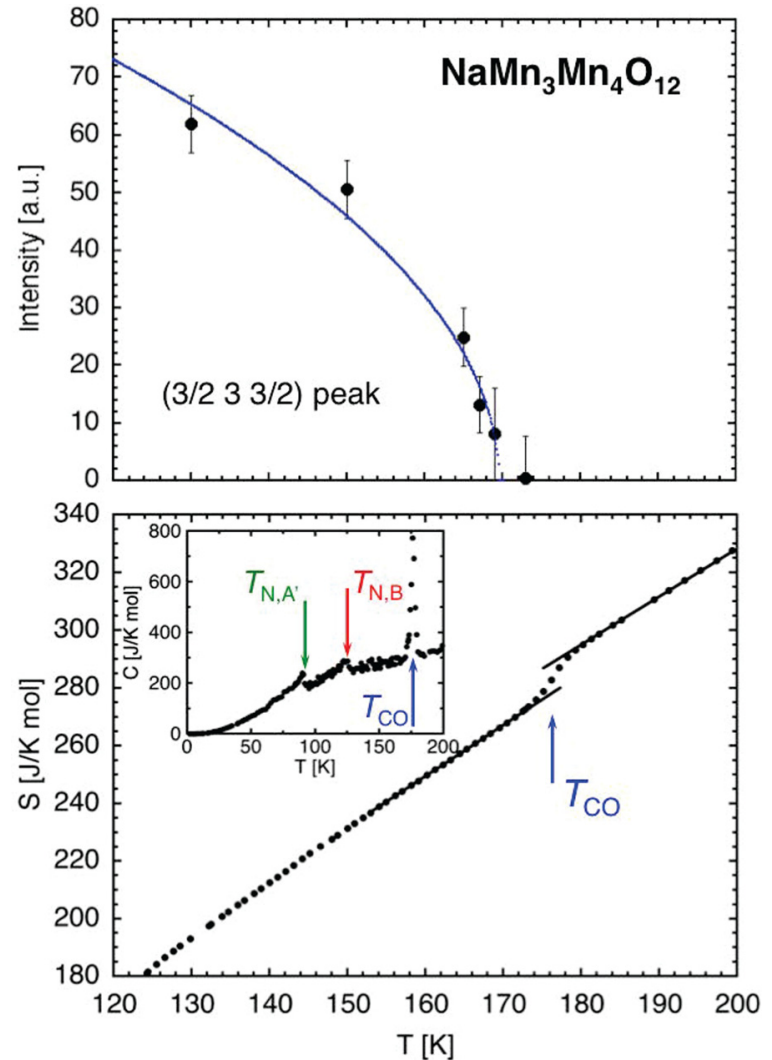

FIG. 4. (Color online) Top: Temperature dependence of the intensity of the $\left(\frac{3}{2}, 3, \frac{3}{2}\right)$ superlattice reflection. The solid line is a meanfield fit described by $I(T) \propto\left(1-T / T_{\mathrm{CO}}\right)^{1 / 2}$. Bottom: Dependence of the specific heat, $C(T)$ (inset), and of the entropy, $S(T)$, obtained by direct integration of the $C(T) / T$ data. Note that the divergence in the $C(T)$ curve at $T_{\mathrm{CO}}$ appears as a jump in the $S(T)$ curve. $T_{N, A^{\prime}}$ and $T_{N, B}$ denote the magnetic orderings of the $A^{\prime}$ and $B$ Mn sublattices. Solid lines are a guide to the eye.

In principle, the modulated structure could be refined by using the above supercell in the $C 2 / \mathrm{m}$ symmetry; in practice, this is not feasible because of the large (27) number of atomic parameters and the difficulty of modeling the asymmetric peak profile. Therefore, we adopted a phenomenological model similar to that proposed by Radaelli et al. [17] for the counterpart compound with a simple perovskite structure $\mathrm{La}_{0.5} \mathrm{Ca}_{0.5} \mathrm{MnO}_{3}$. The distortion pattern, shown in Fig. 5(a), consists of a transverse displacement $\delta$ of the $\mathrm{Mn}^{4+} \mathrm{O}_{6}$ octahedra in the $a c$ plane along the direction perpendicular to q. The displacements are assumed to be rigid because the above octahedra are not Jahn-Teller active. This leads to a shear displacement of the rows of these octahedra along opposite directions perpendicular to q. The displacement leads to the aforementioned zigzag pattern of elongated $\mathrm{Mn}^{3+} \mathrm{O}_{6}$ octahedra. In order to take into account the displacement of the $\mathrm{Mn} A^{\prime}$ ions, it is noted that, while the $\mathrm{Na}(A)-\mathrm{O}$ bond is ionic and isotropic due to the icosahedral coordination, the $\operatorname{Mn}\left(A^{\prime}\right)-\mathrm{O}$ bond has a semicovalent character and the $\operatorname{Mn}\left(A^{\prime}\right)$ ion connecting four octahedral corners forms a square-shaped plaquette. We assume the displacement of the plaquettes to be rigid because a nonplanar coordination of the $\mathrm{Mn}^{3+}$ ion is not favored energetically due to the occupation of the $t_{2 g}$ orbitals.
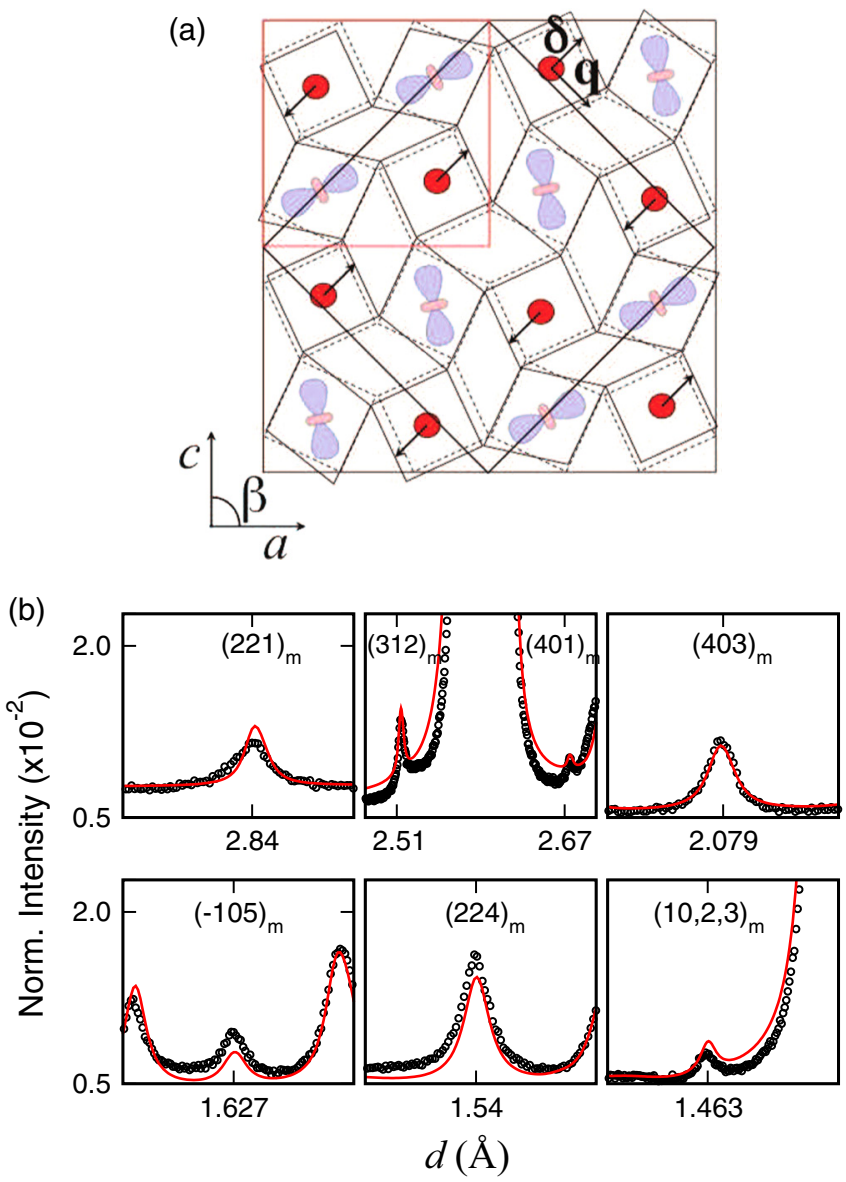

FIG. 5. (Color online) (a) Model of structural modulation in the charge-ordered phase. The small red square on the top left corner indicates the undistorted cubic $\operatorname{Im} \overline{3}$ cell. The large black square rotated by $45^{\circ}$ indicates the supercell. The undistorted and distorted octahedra are sketched by using dashed and solid black lines, respectively. Arrows indicate the shear displacement by $\delta$ of the rows of $\mathrm{Mn}^{4+} \mathrm{O}_{6}$ octahedra along the direction perpendicular to the propagation vector $\mathbf{q}$. The $e_{g} 3 z^{2}-r^{2}$ orbitals in the elongated $\mathrm{Mn}^{3+} \mathrm{O}_{6}$ octahedra and the $\mathrm{Mn}^{4+}$ ions (red circles) are schematically shown. (b) Agreement of the refined model with the strongest nonoverlapping superlattice reflections. Intensities are normalized to the most intense Bragg peak; indices refer to the $C 2 / \mathrm{m}$ monoclinic cell.

Thus, the coordinated displacement of the plaquettes and of the octahedra takes place in an accordion-plaited fashion.

The above model was successfully refined by employing the modulated structure description implemented within the FULLPROF package [18]. The refinement converged to a value $\delta=0.06730(55) \AA$ with a reliability factor $R \approx 6.3$. In Fig. 5(b), note the good agreement for the superlattice reflections. By using the FINDSYM program [19], the symmetry of the refined modulated structure was determined to be $C 2 / \mathrm{m}$, with $a=10.35735(15), b=7.19562(11), c=10.45472(5)$, and $\beta=90.0206(11)^{\circ}$. In the modulated structure, the octahedral $\mathrm{Mn}(B)$ site splits into one $\mathrm{Mn}^{4+}$ site with an $8 j$ general position and two $\mathrm{Mn}^{3+}$ sites $(4 e-4 f)$. The analysis of the refined Mn-O bond lengths (see Table I) shows that the staggered distribution of the long $\mathrm{Mn}-\mathrm{O}$ bonds in the distorted octahedra $\operatorname{Mn}(B 1)$ and $\operatorname{Mn}(B 2)$ are consistent not only with 
TABLE I. Mn-O bond distances for the three Mn B1-3 octahedral sites shown in Fig. 1 resulting from the $C 2 / \mathrm{m}$ refined modulated structure at $T=4.2 \mathrm{~K}$. We also report the average distances, the bond valence sum (BVS), and the distortion parameter, $\Delta=$ $\frac{1}{6} \sum_{i=1}^{6}\left|\frac{d_{\mathrm{MnO}_{i}}-\left\langle d_{\mathrm{MnO}}\right\rangle}{\left\langle d_{\mathrm{MnO}}\right\rangle}\right|^{2}$, for these sites.

\begin{tabular}{lccc}
\hline \hline Mn $B$ site & $\operatorname{Mn}(B 1)$ & $\operatorname{Mn}(B 2)$ & $\operatorname{Mn}(B 3)$ \\
\hline$d(\mathrm{Mn}-\mathrm{O})(\AA)$ & 1.922 & 1.922 & 1.929 \\
& 1.922 & 1.922 & 1.929 \\
& 2.114 & 1.955 & 1.883 \\
& 2.114 & 1.955 & 1.883 \\
& 1.974 & 2.067 & 1.900 \\
$\langle d(\mathrm{Mn}-\mathrm{O})\rangle(\AA)$ & 1.974 & 2.067 & 1.900 \\
$\mathrm{BVS}$ & 2.003 & 1.981 & 1.904 \\
$\Delta\left(\times 10^{-4}\right)$ & 3.18 & 3.34 & 3.99 \\
\hline \hline
\end{tabular}

the CE type of magnetic order, but also with the picture of static charge disproportionation stabilized by the superexchange interaction, in agreement with the nonmodulated structure proposed earlier. Indeed, a bond-valence-sum analysis confirms that the valence of the undistorted $\mathrm{Mn}$ site is $4+$, while that of the distorted Mn sites is 3.18 and 3.34, respectively, similar to the values obtained previously for the average structure. The $\mathrm{MnO}_{4}$ plaquette sites remain in square-planar coordination, with $d(\mathrm{Mn}-\mathrm{O})=1.865,1.919$, and $1.953 \AA$, respectively. Note that the present structural model basically coincides with the aforementioned $a b$ initio calculations [13]. Alternative models, such as the Zener-polaron model proposed earlier for manganites at 0.5 doping [20], which considers the ordering of Mn dimers formed by the double exchange interaction, are not consistent with the present results.

The picture of static charge disproportionation is further supported by a specific heat study as a function of temperature. For these measurements, we used a MagLab microcalorimeter (Oxford Instruments) that employs a thermal relaxation method. In order to exclude any effects arising from granularity or secondary phases, the measurements have been carried out on a bunch of small single crystals. Figure 4(b) shows the specific heat data in the vicinity of the $\mathrm{CO}$ transition and the corresponding entropy difference $\Delta S_{\mathrm{CO}}$. Owing to the negligible latent heat at the transition, $\Delta S_{\mathrm{CO}}$ was obtained by a straightforward integration of the specific heat data, as described in Ref. [21] for the doped perovskite $\mathrm{Pr}_{0.4} \mathrm{Ca}_{0.6} \mathrm{MnO}_{3}$. From Fig. 4, in the present case, we find $\Delta S_{\mathrm{CO}}=11 \pm 1 \mathrm{~mJ} \mathrm{~K}^{-1} \mathrm{~mol}^{-1}$, comparable to the value $4 R \ln 2=23 \mathrm{~mJ} \mathrm{~K}^{-1} \mathrm{~mol}^{-1}$ expected for an ideal order-disorder phase transition of an equal proportion of $\mathrm{Mn}^{3+}$ and $\mathrm{Mn}^{4+}$ ions within a square lattice containing four sites, which corresponds to the $A A_{3}^{\prime} B_{4} \mathrm{O}_{12}$ formula unit. In $\mathrm{Pr}_{0.4} \mathrm{Ca}_{0.6} \mathrm{MnO}_{3}$, a value $40 \%$ smaller was obtained [21], which indicates that the charge ordering is less pronounced in this doped manganite.

The present results may be compared with those reported on $\left(\mathrm{CaMn}_{3}\right) \mathrm{Mn}_{4} \mathrm{O}_{12}$ [22-25] that also displays at $T_{\mathrm{OO}}=$ $250 \mathrm{~K}$ a modulation of a rhombohedrally distorted structure concomitant to a $3: 1$ ordering of the $\mathrm{Mn}^{3+} / \mathrm{Mn}^{4+} B$ ions. The nonmodulated structure was also reported to contain an apically compressed $\mathrm{Mn}^{3+}$ site [23]. However, contrary to the present case, in $\left(\mathrm{CaMn}_{3}\right) \mathrm{Mn}_{4} \mathrm{O}_{12}, T_{\mathrm{OO}}$ is much lower than $T_{\mathrm{JT}}=440 \mathrm{~K}$ at which the Jahn-Teller-driven distortion occurs. Moreover, the modulation is incommensurate and of spiral type. Further theoretical studies may account for the unique type of commensurate and full charge ordering in $\left(\mathrm{NaMn}_{3}\right) \mathrm{Mn}_{4} \mathrm{O}_{12}$. We argue that the existence of two distinct superexchange interaction paths, $A^{\prime}-\mathrm{O}-B$ and $B-\mathrm{O}-B$, with unusually small angles, $\psi \approx 110^{\circ}$ and $\approx 135^{\circ}$, respectively, is expected to enhance the next-nearest-neighbor interactions, as in $\mathrm{TbMnO}_{3}$ [26].

In conclusion, by means of a high-resolution structural study, we have given experimental evidence for a commensurate structural modulation that consistently accounts for the simultaneous $\mathrm{CO} / \mathrm{OO}$ ordering in $\left(\mathrm{NaMn}_{3}\right) \mathrm{Mn}_{4} \mathrm{O}_{12}$ within the framework of the GKA scheme. The unique properties of the above $\mathrm{CO} / \mathrm{OO}$ ordered phase are (i) the commensurability of the structural modulation described by the same propagation vector of the $\mathrm{CE}$ magnetic order, (ii) the almost full static $\mathrm{Mn}^{3+} / \mathrm{Mn}^{4+}$ charge disproportionation, and (iii) the coincidence of the CO/OO ordering with the Jahn-Teller distortion of the $\mathrm{Mn}^{3+}$ site. These properties are ascribed to the absence of disorder caused by chemical substitutions or oxygen defects, which rules out the role of the double exchange interaction, contrary to the case of $R_{0.5} \mathrm{Ca}_{0.5} \mathrm{MnO}_{3}$ [27]. This simple phenomenology should foster theoretical studies that may convincingly account for the stability of the above $\mathrm{CO} / \mathrm{OO}$ orderings and for the functional properties hitherto reported. For instance, it would be interesting to unveil the link between the unusually large tilt of the octahedral network with the enhanced improper ferroelectricity found in the related compound $\left(\mathrm{CaMn}_{3}\right) \mathrm{Mn}_{4} \mathrm{O}_{12}[7,8]$.

We thank G. Calestani, C. Mazzoli, B. Patterson, N. J. Perks, and P. G. Radaelli for useful discussions. A.P. acknowledges financial support from the Fondazione A. Della Riccia and from the Paul Scherrer Institut.
[1] A. Prodi, E. Gilioli, A. Gauzzi, F. Licci, M. Marezio, F. Bolzoni, Q. Huang, A. Santoro, and J. W. Lynn, Nat. Mater. 3, 48 (2004).

[2] A. Prodi, E. Gilioli, R. Cabassi, F. Bolzoni, F. Licci, Q. Huang, J. W. Lynn, M. Affronte, A. Gauzzi, and M. Marezio, Phys. Rev. B 79, 085105 (2009).
[3] K. Takata, I. Yamada, M. Azuma, M. Takano, and Y. Shimakawa, Phys. Rev. B 76, 024429 (2007),

[4] M. Subramanian and A. W. Sleight, Solid State Sci. 4, 347 (2002).

[5] L. Wu, Y. Zhu, S. Park, S. Shapiro, G. Shirane, and J. Tafto, Phys. Rev. B 71, 014118 (2005). 
[6] R. Cabassi, F. Bolzoni, A. Gauzzi, E. Gilioli, A. Prodi, and F. Licci, Phys. Rev. B 74, 045212 (2006).

[7] G. Zhang, S. Dong, Z. Yan, Y. Guo, Q. Zhang, S. Yunoki, E. Dagotto, and J.-M. Liu, Phys. Rev. B 84, 174413 (2011).

[8] R. D. Johnson, L. C. Chapon, D. D. Khalyavin, P. Manuel, P. G. Radaelli, and C. Martin, Phys. Rev. Lett. 108, 067201 (2012).

[9] A. Gauzzi, G. Rousse, F. Mezzadri, G. L. Calestani, G. André, F. Bourée, M. Calicchio, E. Gilioli, R. Cabassi, F. Bolzoni et al., J. Appl. Phys. 113, 043920 (2013).

[10] E. O. Wollan and W. C. Koehler, Phys. Rev. 100, 545 (1955).

[11] M. Marezio, P. D. Dernier, J. Chenavas, and J. C. Joubert, J. Solid State Chem. 6, 16 (1973).

[12] J. Kanamori, J. Phys. Chem. Solids 10, 87 (1959).

[13] S. V. Streltsov and D. I. Khomskii, Phys. Rev. B 89, 201115 (2014).

[14] E. Gilioli, G. Calestani, F. Licci, A. Gauzzi, F. Bolzoni, A. Prodi, and M. Marezio, Solid State Sci. 7, 746 (2005).

[15] F. Gozzo, B. Schmitt, T. Bortolamedi, C. Giannini, A. Guagliardi, M. Lange, D. Meister, D. Maden, P. Willmott, and B. D. Patterson, J. Alloy Compd. 362, 206 (2004).

[16] C. J. Howard and H. T. Stokes, Acta Crystallogr., Sect. B 54, 782 (1998).
[17] P. G. Radaelli, D. E. Cox, M. Marezio, and S.-W. Cheong, Phys. Rev. B 55, 3015 (1997).

[18] J. Rodriguez-Carvajal, Physica B 192, 55 (1993).

[19] H. T. Stokes and D. M. Hatch, J. Appl. Crystallogr. 38, 237 (2005).

[20] A. Daoud-Aladine, J. Rodríguez-Carvajal, L. Pinsard-Gaudart, M. T. Fernández-Díaz, and A. Revcolevschi, Phys. Rev. Lett. 89, 097205 (2002).

[21] K. S. Nagapriya, A. K. Raychaudhuri, B. Bansal, V. Venkataraman, S. Parashar, and C. N. R. Rao, Phys. Rev. B 71, 024426 (2005).

[22] B. Bochu, J. Chenavas, J. C. Joubert, and M. Marezio, J. Solid State Chem. 11, 88 (1974).

[23] B. Bochu, J. L. Buevoz, J. Chenavas, A. Collomb, J. C. Joubert, and M. Marezio, Solid State Commun. 36, 133 (1980).

[24] R. Przenioslo, I. Sosnowska, E. Suard, A. Hewat, and A. N. Fitch, Physica B 344, 358 (2004).

[25] N. J. Perks, R. D. Johnson, C. Martin, L. C. Chapon, and P. G. Radaelli, Nat. Commun. 3, 1277 (2012).

[26] I. A. Sergienko, C. Şen, and E. Dagotto, Phys. Rev. Lett. 97, 227204 (2006).

[27] J. van den Brink, G. Khaliullin, and D. Khomskii, Phys. Rev. Lett. 83, 5118 (1999). 